\title{
Correlation between tumor angiogenesis and invasiveness in thymic epithelial tumors
}

\author{
Masaki Tomita, MD \\ Yasunori Matsuzaki, MD \\ Masao Edagawa, MD \\ Masayuki Maeda, MD \\ Tetsuya Shimizu, MD \\ Masaki Hara, MD \\ Toshio Onitsuka, MD
}

From the Department of Surgery II, Miyazaki Medical College, Kiyotake, Miyazaki, Japan.

Received for publication Aug 16, 2001; revisions requested Nov 28, 2001; revisions received Feb 1, 2002; accepted for publication Feb 6, 2002.

Address for reprints: Masaki Tomita, MD, $\mathrm{PhD}$, Kihara 5200, Kiyotake, Miyazaki, 889-1692,Japan (E-mail: mtomita@ post. miyazaki-med.ac.jp).

J Thorac Cardiovasc Surg 2002;124:493-8

Copyright ( $(2) 2002$ by The American Association for Thoracic Surgery

$0022-5223 / 2002 \$ 35.00+0 \quad \mathbf{1 2 / 1 / 1 2 4 3 8 9}$

doi: $10.1067 / \mathrm{mtc} .2002 .124389$
Objective: Because it is difficult to predict the behavior of thymomas on the basis of morphology alone, other methods for determining tumor aggressiveness must be explored. This study investigated the correlation between angiogenic grade and invasiveness in thymic epithelial tumors.

Methods: Immunohistochemical studies of 46 surgically resected thymic epithelial tumors (18 noninvasive thymomas, 20 invasive thymomas, and 8 thymic carcinomas) were conducted. To highlight the microvessels, we used a specific monoclonal antibody against factor VIII. Expression of vascular endothelial growth factor and basic fibroblast growth factor was determined by using polyclonal antibodies.

Results: Mean microvessel density readings for noninvasive thymomas, invasive thymomas, and thymic carcinomas were $4.6 \pm 3.2,12.4 \pm 7.5$, and $34.4 \pm 16.7$, respectively. Stages I, II, III, and IV of thymoma had microvessel density readings of $4.6 \pm 3.2,8.5 \pm 4.3,13.8 \pm 7.7$, and $22.0 \pm 6.8$, respectively. These findings suggest a significant correlation between microvessel density and tumor invasiveness. Vascular endothelial growth factor expression in noninvasive thymomas, invasive thymomas, and thymic carcinomas was present in $1(5.6 \%)$ of 18,11 $(55.0 \%)$ of 20 , and $5(62.5 \%)$ of 8 patients, respectively, thereby indicating a distinct association between vascular endothelial growth factor expression and increased microvessel density. Basic fibroblast growth factor expression was present in only 8 patients.

Conclusions: In patients with thymic epithelial tumors, there appears to be a significant correlation between tumor angiogenesis and invasiveness. Furthermore, our data suggests that this angiogenesis in thymic epithelial tumors might be, at least in part, dependent on vascular endothelial growth factor expression.

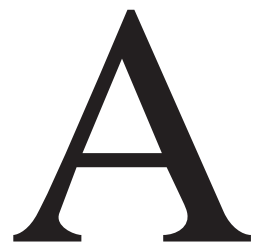

lthough thymic carcinomas show typical features of malignancy, thymomas are more difficult to classify. There are 2 groups: noninvasive thymomas and invasive thymomas. ${ }^{1}$ Cytologically, these groups are unremarkable, making it difficult to distinguish between them. The majority of thymomas are encapsulated tumors that behave in a benign fashion and are therefore not invasive. Even though a thymoma occasionally invades surrounding structures or even metastasizes to thoracic or extrathoracic organs, it retains its cytologically benign features. It is therefore difficult to predict the behavior of thymomas on the basis of morphology alone. ${ }^{2-4}$ However, tumor invasiveness is regarded as the most important factor affecting survival in thymoma. ${ }^{5}$ Therefore the most important 
TABLE 1. Clinical characteristics

\begin{tabular}{lccc}
\hline & $\begin{array}{c}\text { Noninvasive } \\
\text { thymoma }\end{array}$ & $\begin{array}{c}\text { Invasive } \\
\text { thymoma }\end{array}$ & $\begin{array}{c}\text { Thymic } \\
\text { carcinoma }\end{array}$ \\
\hline $\begin{array}{l}\text { Sex } \\
\text { Male }\end{array}$ & 5 & 10 & 2 \\
$\quad \begin{array}{l}\text { Female } \\
\text { Stage } \\
\text { I }\end{array}$ & 13 & 10 & 6 \\
II & 18 & - & - \\
III & - & 10 & - \\
IV & - & 7 & - \\
Myasthenia gravis & - & 3 & - \\
$\quad+$ & & 7 & 0 \\
- & 7 & 13 & 8 \\
Pure red cell aplasia & 11 & 1 & 0 \\
$\quad+$ & 1 & 19 & 8 \\
- & 17 & & \\
\hline
\end{tabular}

prognostic factor for thymomas is their macroscopic appearance at the operation, and Masaoka staging (I-IV) is useful in staging patients with thymoma. ${ }^{5}$

Previous studies have linked tumor aggressiveness to Ki67, ${ }^{6}$ epidermal growth factor receptor, ${ }^{6}$ bcl-2, ${ }^{7}$ argyrophilic nucleolar organizer region, ${ }^{8}$ and matrix metalloproteinase 2 activation $^{9}$ in thymic epithelial tumors.

Because the functional relationship between tumor-induced angiogenesis and tumor invasion is generally accepted with regard to cancerous tissue, ${ }^{10}$ we hypothesized that there might be a correlation between angiogenic grade and invasiveness in thymic epithelial tumors. To prove this hypothesis, we investigated tumor angiogenesis and also assessed the expression of vascular endothelial growth factor (VEGF) and basic fibroblast growth factor (bFGF), pivotal mediators of tumor angiogenesis.

\section{Materials and Methods}

\section{Patients and Tissue Samples}

Forty-six consecutive patients (17 men and 29 women) with thymic epithelial tumors who were treated surgically at Miyazaki Medical College were included in this study. Patient ages ranged from 19 to 77 years (mean, 55.2 years). The Masaoka staging system $^{5}$ was followed to assign postoperative staging of thymomas. Thymomas were divided into 2 groups on the basis of surgical and histologic findings as follows: noninvasive thymoma, clinical stage I; invasive thymoma, clinical stages II, III, or IV. This classification was mainly done on the basis of the macroscopic appearance of the tumor at the time of operations by 2 or more surgeons. The tumors included 18 noninvasive thymomas, 20 invasive thymomas, and 8 thymic carcinomas. Fourteen patients ( 7 with noninvasive and 7 with invasive thymomas) had concomitant myasthenia gravis (MG), and 2 patients ( 1 with noninvasive and 1 with invasive thymomas) had pure red cell aplasia. Table 1 summarizes the clinical characteristics of these patients. Surgically resected tissue samples previously fixed in formalin and embedded in paraffin were used in this study.

\section{Immunohistochemical Studies}

To highlight the microvessels, we used a specific monoclonal antibody against factor VIII (Dako Diagnostika, Hamburg, Germany). To determine VEGF and bFGF expression, we used polyclonal antibodies against VEGF (Santa Cruz Biotechnology, Santa Cruz, Calif) and bFGF (Santa Cruz Biotechnology). Before staining, serial $4-\mu \mathrm{m}$-thick sections were pretreated with microwave heating in $10 \mathrm{mmol} / \mathrm{L}$ citric acid buffer for $4 \times 5$ minutes. These sections were immersed in $0.6 \% \mathrm{H}_{2} \mathrm{O}_{2}$ in methanol for 20 minutes at room temperature to block endogenous peroxidase activity. After blocking nonspecific protein binding with an overnight incubation with Block Ace (Dainippon, Inc, Osaka, Japan), the sections were incubated with primary antibodies against human factor VIII (1:200), VEGF (1:100), and bFGF (1:100), respectively, at $4{ }^{\circ} \mathrm{C}$ overnight. Subsequently, sections were incubated with the secondary antiserum (1:500) for 1 hour, followed by an incubation with peroxidase-antiperoxidase complex for 30 minutes at room temperature. The sections were visualized with a diaminobenzidine/metal concentration $(10 \times)$ and stable peroxide substrate buffer $(1 \times)$ system (Pierce, Rockford, Ill). The sections were then washed with water and counterstained with hematoxylin. Immunohistologic results were assessed semiquantitatively by 2 authors. Microvessel density was determined, as described by Weidner and colleagues, ${ }^{11}$ in the area of the most intense vascularization (hot spot) of each tumor, and the average count was recorded. VEGF expression was evaluated as described in our previous study. ${ }^{12}$ Tumors in which greater than $30 \%$ of the tumor cells stained more intensely than normal smooth muscle were judged to be VEGF positive. Basic FGF antibody reactivity with individual tissue sections was considered positive if unequivocal staining of the membrane or cytoplasm was observed. ${ }^{13}$

\section{Statistical Analysis}

Data for the groups were compared by using the 2-tailed independent Student $t$ test. Correlation trends were assessed by using the Spearman rank correlation coefficient.

\section{Results}

\section{Microvessel Count}

At a magnification of $200 \times$, microvessels appeared as brown linear fragments; occasionally, a nucleus was visible. Tiny lumens within these microvessels often resembled small circular or fusiform structures (Figure 1). In noninvasive thymomas (group NT) microvessels were predominantly observed in small groups at the margin of the tumor and rarely seen in the tumor parenchyma. In invasive thymomas (group IT) and thymic carcinomas (group TC), however, microvessels were present in the tumor parenchyma in addition to those observed at the margin. The number (mean \pm SD) of microvessels (per $200 \times$ field) in groups NT, IT, and TC was $4.6 \pm 3.2,12.4 \pm 7.5$, and $34.4 \pm 16.7$, respectively. As shown in Figure 2, these data demonstrate a significant correlation between microvessel density and invasiveness (Spearman rank correlation: $r$ value $=0.793, P<.0001)$.

The number (mean \pm SD) of microvessels (per $200 \times$ 

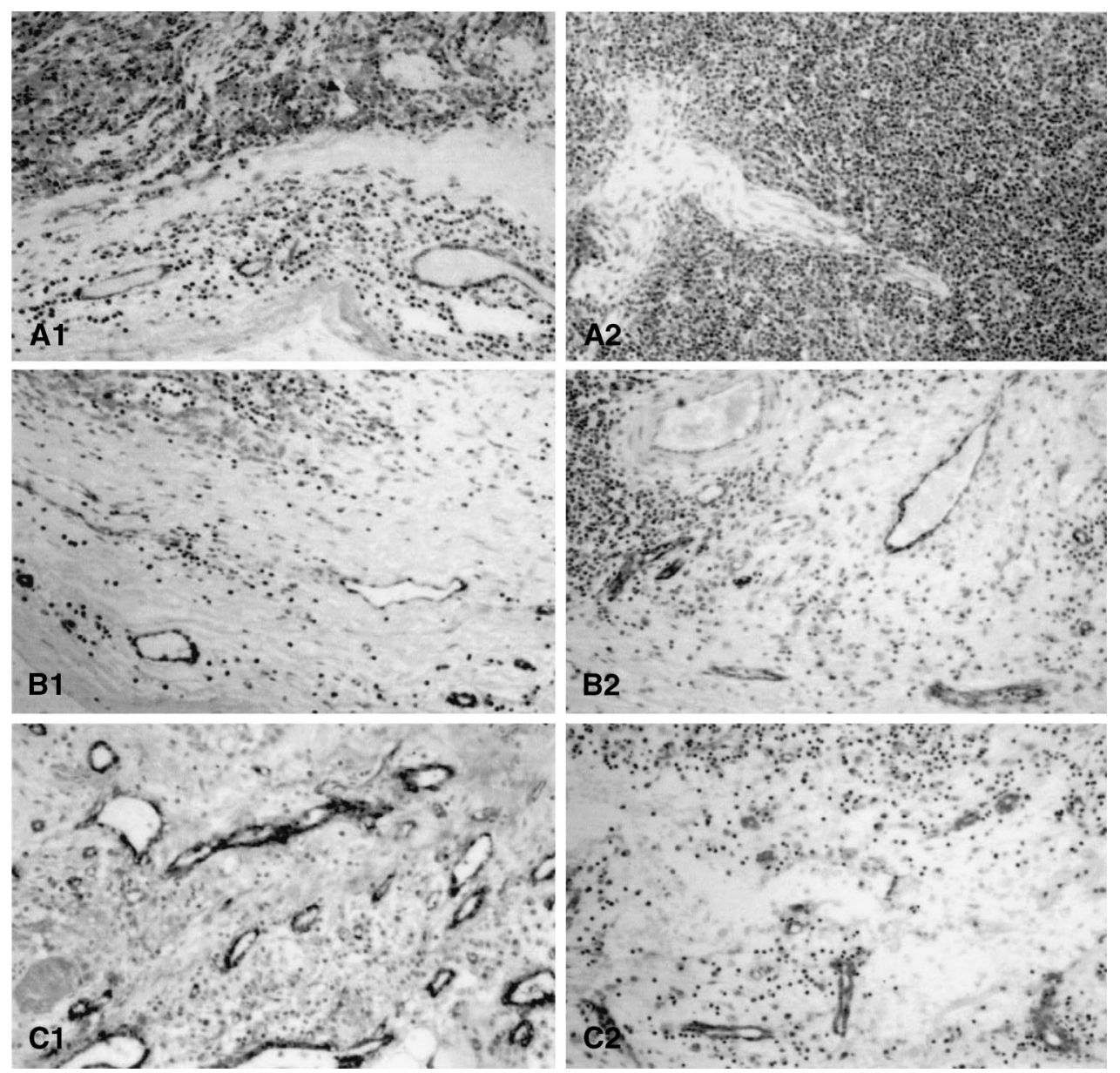

Figure 1. Immunohistochemical staining of microvessels in noninvasive thymoma (A1 and A2), invasive thymoma (B1 and B2), and thymic carcinoma (C1 and C2). Microvessels were observed at the edge of the tumor (A1, B1, and C1). Microvessels were also seen in the parenchyma of invasive thymoma (B2) and thymic carcinoma (C2) but not in noninvasive thymoma (A2). (Original magnification $100 \times$.)

field) of thymomas in stages I, II, III, and IV (as determined with the Masaoka staging system) was $4.6 \pm 3.2,8.5 \pm 4.3$, $13.1 \pm 7.7$, and $22.0 \pm 6.8$, respectively. These data (Figure 3) suggest a definite correlation between microvessel density and clinical stage of thymomas (Spearman rank correlation: $r$ value $=0.727, P<.0001)$.

The microvessel count (per 200 $\times$ field, mean \pm SD) in patients with MG was $8.0 \pm 5.2$; in patients without the disease, the count was $14.0 \pm 14.9$. These results suggest an inverse association between the presence of MG and microvessel density $(P=.019)$. We did not demonstrate any correlation between the presence of pure red cell aplasia and microvessel density $(P=.75)$.

\section{VEGF and bFGF Expression}

VEGF-positive staining was predominantly observed in the cytoplasm of the tumor cells. VEGF expression in groups NT, IT, and TC was $1(5.6 \%)$ of $18,11(55.0 \%)$ of 20 , and
$5(62.5 \%)$ of 8 patients, respectively. These results indicate a definite correlation between VEGF expression and invasiveness (Spearman rank correlation: $r$ value $=0.672, P<$ .0001). Furthermore, as shown in Figure 4, our data suggest an association between VEGF expression and microvessel density $(P=.003)$.

Basic FGF immunoreactivity was also shown to be cytoplasmic. Because, in the present study, bFGF expression was detected in only 8 patients ( 0 in group NT, 5 in group IT, and 3 in group TC), the relationship between bFGF expression and thymic tumor invasiveness was not evaluated. We did, however, demonstrate a correlation between bFGF expression and microvessel density $(P=.04$, data not shown).

\section{Discussion}

Distinguishing between noninvasive and invasive thymomas by means of histologic examination alone is difficult 


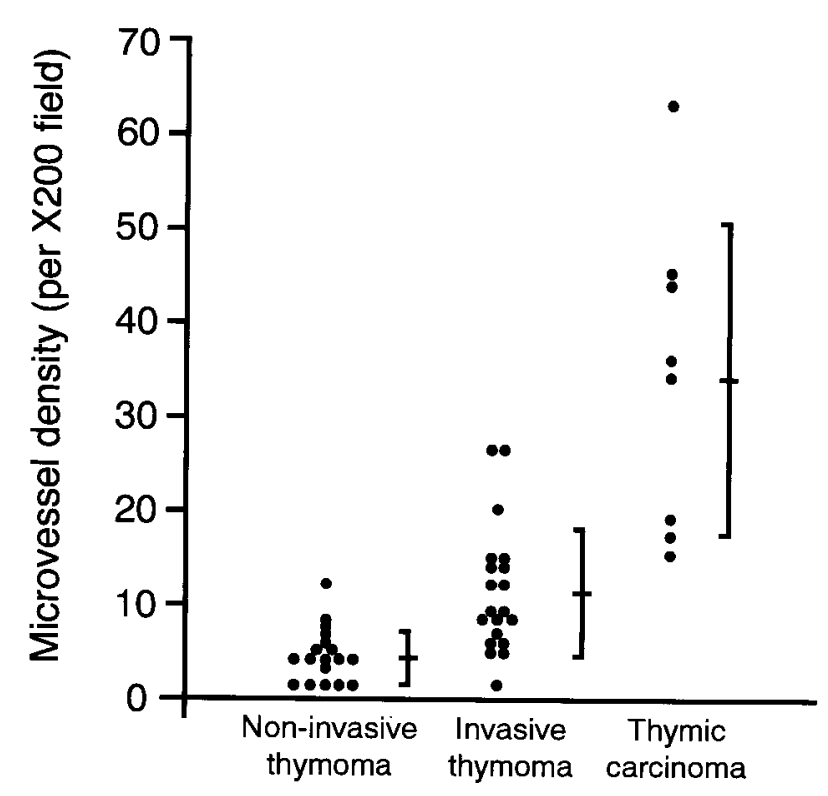

Figure 2. Microvessel density in noninvasive thymoma, invasive thymoma, and thymic carcinoma (Spearman rank correlation: $r$ value $=0.793, P<.0001$ ).

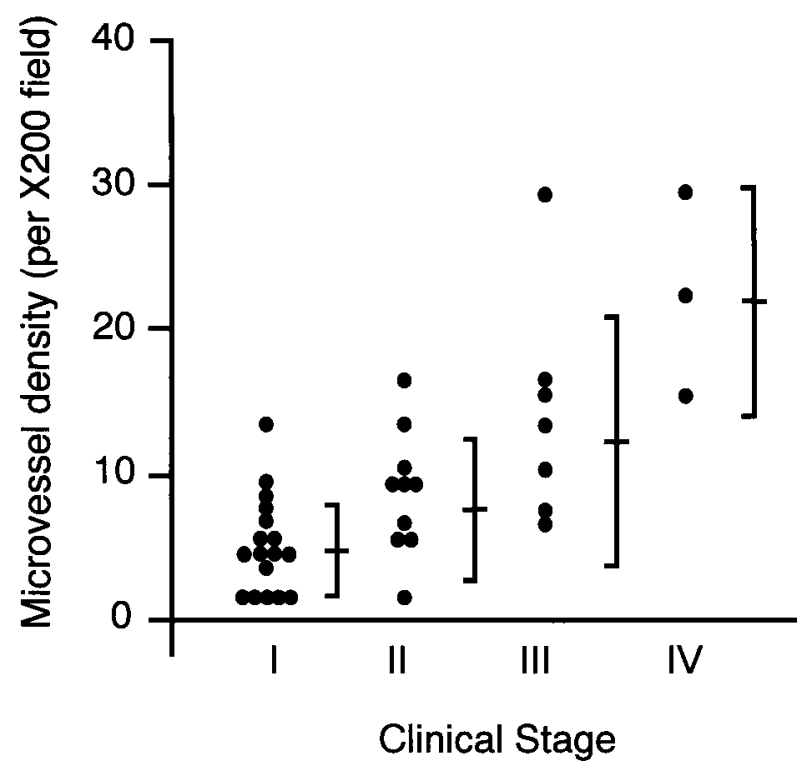

Figure 3. Relationship between microvessel density and clinical stage in thymoma. (Spearman rank correlation: $r$ value $=0.727$, $\boldsymbol{P}<$.0001).

because the morphology of the 2 types does not differ significantly. The most important prognostic indicator is the macroscopic appearance of the thymoma at the time of the operation (ie, irrespective of whether it is encapsulated). ${ }^{14,15}$

Because angiogenesis is essential for tumor growth, ${ }^{16}$ an

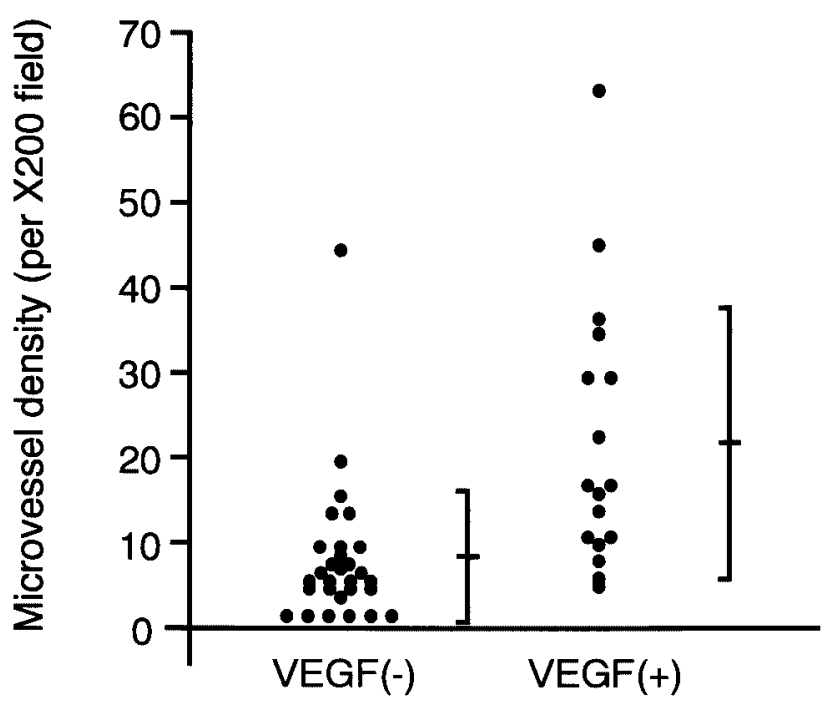

Figure 4. Relationship between microvessel density and VEGF expression $(P=.003)$.

enhanced vascular supply could reflect a malignant potential. Blood vessels might play an important role in tumorogenesis by supplying nutrients and oxygen and disposing of metabolic waste products, thereby allowing tumor invasion. Although numerous studies regarding tumor angiogenesis in human cancers have been published, they do not address angiogenesis in thymic epithelial tumors. In our study we assessed the relationship between angiogenic grade and invasiveness in thymic epithelial tumors. Our data indicate a significant correlation between tumor angiogenesis and invasiveness in thymic epithelial tumors. Thymic carcinomas are different types of tumors than thymomas, and they have more aggressive histologic appearances and clinical outcome than those of thymomas. It has been unknown whether angiogenesis of thymic carcinomas is more aggressive than that of thymomas. Therefore we included thymic carcinoma and found that thymic carcinomas have more aggressive angiogenic function than thymomas. In addition, we demonstrated the presence of parenchymal microvessels in invasive thymoma but not in noninvasive thymoma. This suggests it might be possible to distinguish between these 2 groups on the basis of the presence or absence of parenchymal microvessels. Further studies in this area are warranted.

It was reported that World Health Organization (WHO) histologic classification reflects the clinical features of thymic epithelial tumors. ${ }^{17}$ Our recent data indicated a trend toward an association between WHO histologic classification and tumor angiogenesis in thymic epithelial tumors; however, it did not reach statistical significance (unpublished data). Whether microvessel density in thymic epithelial tumor is related to WHO histologic classification warrants further investigation. 
Angiogenesis is controlled by angiogenic factors that regulate the coordinated extracellular matrix remodeling, endothelial cell proliferation, capillary differentiation, and anastomosis necessary to establish a blood supply. ${ }^{18,19}$ Matrix metalloproteinases, the main function of which is to degrade extracellular matrix, are thought to play a role not only in tumor invasion but also in angiogenesis. ${ }^{20}$ Although factors that induce tumor angiogenesis in thymic epithelial tumors have not been clearly defined, Kondo and coworkers ${ }^{9}$ reported a correlation between matrix metalloproteinase 2 activation and invasiveness in thymic epithelial tumors. This study suggests that matrix metalloproteinase 2 might be an inducer of angiogenesis in these types of tumors.

VEGF, a specific mitogen for endothelium, increases vascular permeability and induces proteolytic enzymes necessary for vascular remodeling. ${ }^{21}$ Our data indicate a definite association between VEGF expression and invasiveness in thymic epithelial tumors. More importantly, because of a strong correlation between VEGF expression and microvessel density, angiogenesis in thymic epithelial tumors might be, at least in part, dependent on VEGF expression.

Basic FGF is also an important angiogenesis inducer. ${ }^{22}$ Although we demonstrated a relationship between bFGF expression and microvessel density, bFGF-positive patients accounted for only 8 (17.4\%) of 46 patients in our study. On the basis of these numbers, it seems likely that angiogenesis of thymic epithelial tumors is less dependent on the presence of bFGF.

Interestingly, although our results showed an inverse association between the presence of MG and microvessel density, the number of patients with MG in group NT was essentially the same as for those in group IT (Table 1). In addition, it has been reported that MG can no longer be considered an adverse prognostic factor in thymoma. ${ }^{23}$ Taken together, the presence of MG might not be indicative of tumor invasiveness and subsequent patient survival. Furthermore, it has been proposed that thymomas associated with MG might be initiated by an immune reaction against a tumor protein related to or cross-reactive with the acetylcholine receptor. ${ }^{24}$ Whether microvessel density in MGassociated thymomas is related to this immune reaction warrants further investigation.

It had been reported that antiangiogenic agents have been emphasized as potential therapeutic agents for patients with malignancies. ${ }^{25}$ Furthermore, it had also been reported that anti-VEGF therapy significantly inhibited the growth of established experimental tumors. ${ }^{26}$ Thus, taken together with our data, antiangiogenic agents might be a new treatment for patients with invasive thymic epithelial tumors.

In conclusion, we found a significant correlation be- tween tumor angiogenesis and invasiveness in thymic epithelial tumors. Our results also suggest that angiogenesis of these types of tumors might be dependent on VEGF expression.

We thank Mrs Yasuko Tobayashi for her skillful technical assistance and Mrs Nancy Folks for her support in preparing this manuscript.

\section{References}

1. Levine GD, Rosai J. Thymic hyperplasia and neoplasia: a review of current concepts. Hum Pathol. 1978;9:495-515.

2. Kornstein MJ. Controversies regarding the pathology of thymomas. Pathol Апnи. 1992;2:1-15.

3. Shimosato Y. Controversies surrounding the subclassification of thymoma. Cancer. 1994;74:542-4.

4. Kornstein MJ, Curran WJ, Turrisi AT, Brooks JJ. Cortical versus medullary thymomas: a useful morphologic distinction? Hum Pathol. 1988;19:1335-9.

5. Masaoka A, Monden Y, Nakahara K, Tanioka T. Follow-up study of thymomas with special reference to their clinical stages. Cancer. 1981;48:2485-92.

6. Gilhus NE, Jones M, Turley H, Gatter KC, Nagvekar N, NewsomDavis $\mathrm{J}$, et al. Oncogene proteins and proliferation antigens in thymomas: increased expression of epidermal growth factor receptor and Ki67 antigen. J Clin Pathol. 1995;48:447-55.

7. Chen FF, Yan JJ, Jin YT, Su IJ. Detection of bcl-2 and p53 in thymoma: expression of bcl-2 as a reliable marker of tumor aggressiveness. Hum Pathol. 1996;27:1089-92.

8. Lee YC, Chern JH, Pan CC, Chang SC, Perng RP. Argyrophilic nucleolar organizer regions in cells of thymoma and thymic carcinoma: correlation with DNA ploidy and clinicopathologic characteristics. Chest. 1999;115:1115-9.

9. Kondo K, Kinoshita H, Ishikura H, Miyoshi T, Hirose T, Matsumori $\mathrm{Y}$, et al. Activation of matrix metalloproteinase-2 is correlated with invasiveness in thymic epithelial tumors. J Surg Oncol. 2001;76:16975.

10. Alessandro R, Kohn EC. Molecular genetics of cancer. Tumor invasion and angiogenesis. Cancer. 1995;76:1874-7.

11. Weidner N, Semple JP, Welch WR, Folkman J. Tumor angiogenesis and metastatic correlation in invasive breast carcinoma. $N$ Engl J Med. 1991;324:1-8

12. Tomita M, Matsuzaki Y, Onitsuka T. Effect of mast cells on tumor angiogenesis in lung cancer. Ann Thorac Surg. 2000;69:1686-90.

13. Takanami I, Tanaka F, Hashizume T, Kikuchi K, Yamamoto Y, Yamamoto T, et al. The basic fibroblast growth factor and its receptor in pulmonary adenocarcinomas: an investigation of their expression as prognostic markers. Eur J Cancer. 1996;32A:1504-9.

14. Lewis JE, Wick MR, Scheithauer BW, Bernatz PE, Taylor WF. Thymoma. A clinicopathologic review. Cancer. 1987;60:2727-43.

15. Maggi G, Giaccone G, Donadio M, Ciuffreda L, Dalesio O, Leria G, et al. Thymomas. A review of 169 cases, with particular reference to results of surgical treatment. Cancer. 1986;58:765-76.

16. Fox SB, Gatter KC, Harris AL. Tumour angiogenesis. J Pathol. 1996;179:232-7.

17. Okumura M, Miyoshi S, Fujii Y, Takeuchi Y, Shiono H, Inoue M, et al. Clinical and functional significance of WHO classification on human thymic epithelial neoplasms: a study of 146 consecutive tumors. Am J Surg Pathol. 2001;25:103-10.

18. Paweletz N, Knierim M. Tumor-related angiogenesis. Crit Rev Oncol Hematol. 1989;9:197-242.

19. Blood CH, Zetter BR. Tumor interactions with the vasculature: angiogenesis and tumor metastasis. Biochim Biophys Acta. 1990;1032: 89-118.

20. John A, Tuszynski G. The role of matrix metalloproteinases in tumor angiogenesis and tumor metastasis. Pathol Oncol Res. 2001;7:14-23.

21. Ferrara N, Davis-Smyth T. The biology of vascular endothelial growth factor. Endocr Rev. 1997;18:4-25. 
22. Rifkin DB, Moscatelli D. Recent developments in the cell biology of basic fibroblast growth factor. J Cell Biol. 1989;109:1-6.

23. Kohman LJ. Controversies in the management of malignant thymoma. Chest. 1997;112:296S-300S.

24. Marx A, Wilisch A, Schultz A, Gattenlohner S, Nenninger R, MüllerHermelink HK. Pathogenesis of myasthenia gravis. Virchows Arch. 1997;430:355-64
25. Yamaoka M, Yamamoto T, Masaki T, Ikeyama S, Sudo K, Fujita T. Inhibition of tumor growth and metastasis of rodent tumors by the angiogenesis inhibitor O-(chloroacetyl-carbamoyl)fumagillol (TNP470; AGM-1470). Cancer Res. 1993;53:4262-7.

26. Im SA, Kim JS, Gomez-Manzano C, Fueyo J, Liu TJ, Cho MS, et al. Inhibition of breast cancer growth in vivo by antiangiogenesis gene therapy with adenovirus-mediated antisense-VEGF. Br J Cancer. 2001;84:1252-7.

\section{Availability of Journal back issues}

As a service to our subscribers, copies of back issues of The Journal of Thoracic and Cardiovascular Surgery for the preceding 5 years are maintained and are available for purchase from Mosby until inventory is depleted. Please write to Mosby, Subscription Customer Service, 6277 Sea Harbor Dr, Orlando, FL 32877, or call $800-654-2452$ or $407-345-4000$ for information on availability of particular issues and prices. 